Gut and Liver, Vol. 12, No. 1, January 2018, pp. 86-93

\title{
The Real-World Safety and Efficacy of Daclatasvir and Asunaprevir for Elderly Patients
}

Shinya Taki ${ }^{1}$, Hideyuki Tamai ${ }^{1}$, Yoshiyuki Ida ${ }^{1}$, Naoki Shingaki ${ }^{1}$, Akira Kawashima $^{2}$, Ryo Shimizu ${ }^{1}$, Kosaku Moribata $^{1}$, Takao Maekita $^{1}$, Mikitaka Iguchi ${ }^{1}$, Jun Kato ${ }^{1}$, Taisei Nakao ${ }^{2}$, and Masayuki Kitano ${ }^{1}$

${ }^{I}$ Second Department of Internal Medicine, Wakayama Medical University, and ${ }^{2}$ Department of Internal Medicine, Naga Municipal Hospital, Wakayama, Japan

Background/Aims: Although daclatasvir with asunaprevir was approved in Japan for interferon ineligible or intolerant patients, patients aged $\geq 75$ years were excluded in the phase III trial. The present study aimed to evaluate the safety and efficacy of this therapy for elderly patients aged $\geq 75$ years and to clarify whether an extremely high sustained virological response (SVR) rate can be achieved, even in a real-world setting when patients with resistance-associated substitutions (RASs) to nonstructural protein 5A (NS5A) inhibitors or prior simeprevir failure are excluded. Methods: Daclatasvir (60 mg) and asunaprevir (100 mg) were orally administered daily for 24 weeks. Patients without pre-existing NS5A RASs and simeprevir failure were enrolled in this study. Results: Overall, 110 patients were treated. The median age was 73 years old. The SVR rates of total patients, those aged $\geq 75$ years, and those aged $<75$ years were 97\% (107/110), 98\% (46/47), and 97\% (61/63), respectively. The treatment of two patients (2\%) was discontinued because of adverse events. Conclusions: Daclatasvir with asunaprevir was a safe treatment, even in patients aged $\geq 75$ years. When patients without pre-existing NS5A RASs and prior simeprevir failure were selected, an extremely high SVR rate could be achieved irrespective of age. (Gut Liver 2018;12:86-93)

Key Words: Hepacivirus; Genotype 1b; Daclatasvir; Asunaprevir

\section{INTRODUCTION}

The goal of therapy for patients infected with hepatitis $C$ virus (HCV) is to prevent liver-related death such as cirrhosis or hepatocellular carcinoma (HCC). Along with advancing age and liver fibrosis, the risk of hepatocarcinogenesis increases. ${ }^{1}$ Therefore, HCV in infected elderly and/or cirrhotic patients should be eradicated as soon as possible. ${ }^{2}$ However, most of these patients are ineligible or intolerant for pegylated interferon (IFN) and ribavirin therapy because of cytopenia, comorbidities and a high risk of serious adverse effects. In clinical practice, even with reduced doses of pegylated IFN and ribavirin, the safety and efficacy for elderly and/or cirrhotic patients was unsatisfactory.,4

In November 2011, the first-generation of nonstructural (NS) 3/4A protease inhibitor, telaprevir-based triple therapy became available for patients in Japan infected with genotype $1 \mathrm{HCV}{ }^{5}$ Two years later, the next triple therapy using second generation protease inhibitor, simeprevir, was introduced. ${ }^{6}$ Although these therapies markedly improved sustained virological response (SVR) rates up to around 70\% to 90\%, patient's ages in these phase III trials were young and cirrhotic patients were excluded. In elderly and/or cirrhotic patients, even a reduced dose of telaprevir-based triple therapy had limited safety and efficacy. ${ }^{7}$

In September 2014, the clinical use of all-oral, interferon- and ribavirin-free, dual direct-acting antiviral combination therapy using nonstructural protein 5A (NS5A) inhibitor daclatasvir and NS3/4A protease inhibitor asunaprevir was first approved in Japan for IFN-ineligible or intolerant patients infected with genotype $1 \mathrm{HCV}$. This therapy was well tolerated and achieved a high SVR rate (85\%) in a Japanese phase III trial. ${ }^{8}$ However, patients aged older than 75 years old were excluded, patients with serious renal dysfunction or patients with a history of HCC treatment were excluded in the phase III trial. To meet these unmet medical needs, real-world evidence is needed.

Furthermore, it was clarified that pre-existing HCV resistanceassociated substitutions (RASs) to NS5A inhibitors were associated with low SVR. ${ }^{8}$ Besides, as RASs profile of asunaprevir is similar to that of simeprevir, it is predictable that this therapy

Correspondence to: Hideyuki Tamai

Second Department of Internal Medicine, Wakayama Medical University, 811-1 Kimiidera, Wakayama 641-0012, Japan

Tel: +81-73-447-2300, Fax: +81-73-445-3616, E-mail: tamahide@wakayama-med.ac.jp

Received on January 25, 2017. Revised on March 22, 2017. Accepted on March 22, 2017. Published online August 14, 2017

pISSN 1976-2283 eISSN 2005-1212 https://doi.org/10.5009/gnl17048

@ This is an Open Access article distributed under the terms of the Creative Commons Attribution Non-Commercial License (http://creativecommons.org/licenses/by-nc/4.0) which permits unrestricted non-commercial use, distribution, and reproduction in any medium, provided the original work is properly cited. 
for patients with failure of simeprevir-based triple therapy would lead to a low SVR rate. Strong RASs to both NS5A drugs and NS3/4A inhibitors have been found after the treatment failure of this therapy. ${ }^{8}$ As the next therapy for them has not yet been established, in order not to make RASs to multidrugs, this therapy should not be applied to patients with factors associated with low SVR, such as pre-existing RASs or prior simeprevir failure.

We conducted a post-marketing prospective cohort study to evaluate the safety and efficacy of daclatasvir plus asunaprevir for elderly patients over 75 years of age. We aimed to clarify whether an extremely high SVR rate can be achieved even in a real-world setting when patients with NS5A RASs and simeprevir failure were excluded.

\section{MATERIALS AND METHODS}

\section{Patients}

The inclusion criterion was adult patients infected with genotype $1 \mathrm{~b} \mathrm{HCV}$, who receive daclatasvir and asunaprevir combination therapy. Exclusion criteria were any of following: (1) being infected with other genotypes other than genotype1b HCV; (2) strongly positive HCV RASs to NS5A inhibitors; (3) pretreatment failure of simeprevir-based triple therapy; (4) decompensated cirrhosis (Child-Pugh class B or C); (5) any form of cancer. Between November 2014 and July 2015, patients who were eligible were enrolled in the present study in the Wakayama Medical University Hospital and Naga Municipal Hospital. Before entry, all patients were informed of the results of RASs, the risk of treatment failure by pre-existing RASs and the absence of the next established therapy for strong RASs to multidrug generated after failure of this treatment. As a general rule, although this therapy was not recommended for patients with weakly positive NS5A RASs, only patients who strongly requested to receive this therapy were enrolled in this study.

Liver cirrhosis was diagnosed clinically by liver biopsy or imaging studies using morphologic signs of cirrhosis from portal hypertension, such as portosystemic shunt or hypersplenism. IFN-ineligible patients were defined as patients with any of the following: ${ }^{2}$ (1) age $\geq 65$ years; (2) white blood cell count $<2,000 /$ $\mathrm{mm}^{3}$; (3) platelet count $<130,000 / \mathrm{mm}^{3}$; (4) hemoglobin level $<13 \mathrm{~g} / \mathrm{dL}$; (5) comorbid disorder such as psychiatric disease, heart disease, cerebrovascular disease, thyroid disease, autoimmune disease or uncontrolled diabetes; or (6) low body weight (<40 kg).

All study protocols were approved by the ethics committees of the participating hospitals. Written informed consent was obtained from all patients included in this study. The present study was registered on the University Hospital Medical Information Network (trial ID: 000023267).

\section{Treatment regimens}

For 24 weeks, daclatasvir (Daklinza; Bristol-Myers Squibb, Tokyo, Japan) was orally administered as a $60 \mathrm{mg}$ tablet once daily and asunaprevir (Sunvepra; Bristol-Myers Squibb) was orally administered as a $100 \mathrm{mg}$ soft gel capsule twice daily.

\section{Laboratory tests}

Patients were examined for pre-existing RASs to NS5A inhibitors and NS3/4 inhibitors by polymerase chain reaction (PCR) invader assay (BML, Tokyo, Japan). Amino acid substitutions resistant to NS3/4 protease inhibitors V36A, T54A/S, Q80L/R, R155K/Q/T, A156S/T/V and D168A/E/H/T/V and resistant to NS5A inhibitors $\mathrm{L} 31 \mathrm{~F} / \mathrm{M} / \mathrm{V}$ and $\mathrm{Y} 93 \mathrm{H}$ were identified. When more than $20 \%$ of variants were detected, RASs were judged as strongly positive. When less than $20 \%$ of variants were detected, they were judged as weakly positive. ${ }^{9}$ The amount of HCV RNA was measured using quantitative RT-PCR (COBAS TaqMan ${ }^{\circledR}$

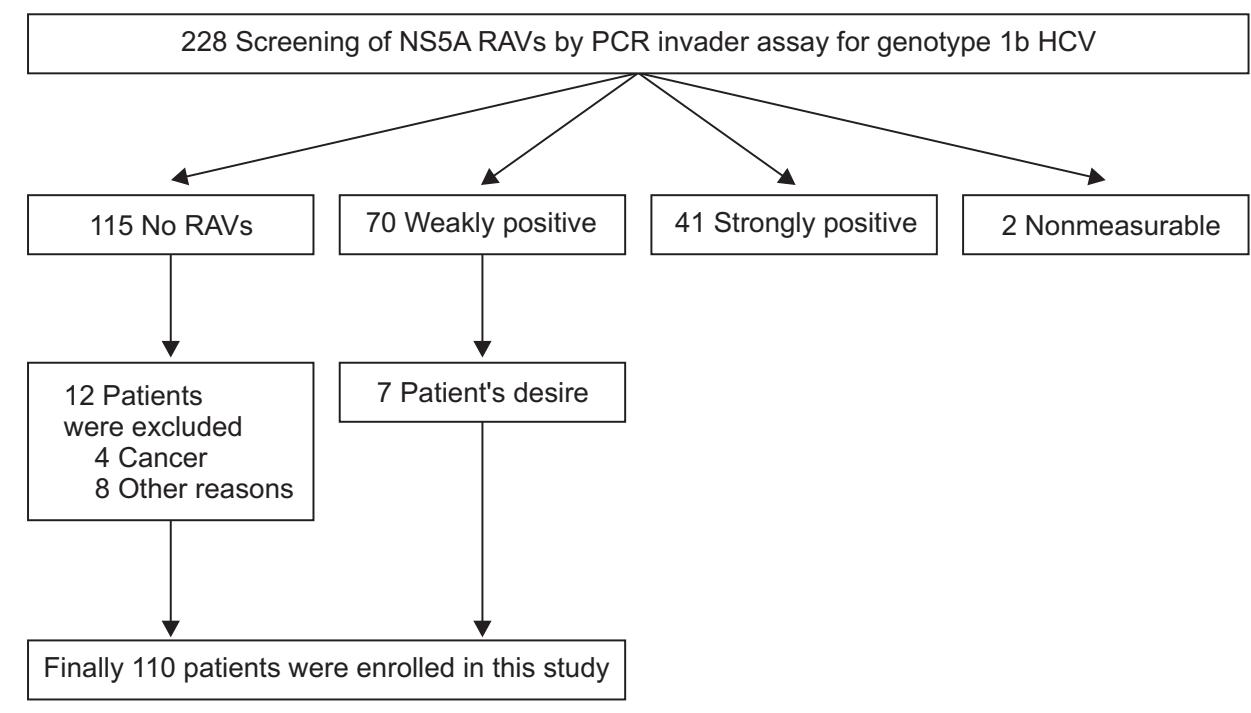

Fig. 1. Flow chart of patients. NS, nonstructural; RAVs, resistance-associated variants; PCR, polymerase chain reaction; $\mathrm{HCV}$, hepatitis C virus. 
PCR assay version 2; Roche Diagnostics, Branchburg, NJ, USA) and were checked on the day of therapy initiation at weeks 1 and 2, as well as every 4 weeks up to 12 weeks after the end of therapy. Serum levels of hyaluronic acid and type IV collagen 7S were measured for assessment of liver fibrosis on the day of therapy initiation. Biochemical analyses including blood counts, serum alanine aminotransferase (ALT), aspartate aminotransferase, $\gamma$-glutamyl transferase ( $\gamma$-GT), estimated glomerular filtration rate (eGFR) and $\alpha$-fetoprotein levels were performed every 4 weeks up to 12 weeks after the end of therapy.

\section{Assessment of effectiveness}

SVR was defined as HCV RNA measured using the TaqMan ${ }^{\circledR}$ PCR assay being negative at the end of therapy and remaining negative for 12 weeks after the end of therapy.

\section{Assessment of safety and tolerability}

Patients were assessed for safety and tolerability during treatment by attending physicians who monitored adverse events and laboratory parameters such as blood cell counts and liver and renal function tests every 2 weeks. Transaminase elevation was assessed according to Common Terminology Criteria for Adverse Events version 4.0 (https://ctep.cancer.gov/protocoldevelopment/electronic_applications/ctc.htm). The incidence of and reasons for therapy discontinuation or interruption due to adverse events were analyzed.

\section{Statistical analysis}

Therapeutic efficacy was evaluated using an intention-totreat analysis. The Mann-Whitney U-test was used to analyze continuous variables. Fisher exact test or the chi-square test were used to analyze categorical variables. Values of $p<0.05$ were considered statistically significant. SPSS statistical software version 21.0J for Windows (SPSS Inc., Tokyo, Japan) was used for data analyses.

\section{RESULTS}

\section{Patients' baseline characteristics}

NS5A RASs of 228 consecutive patients with genotype $1 \mathrm{~b}$ HCV were measured by PCR invader assay (BML). The patients' flow chart is shown in Fig. 1. The prevalence of NS5A RASs was 18\% (41/228). Finally, 110 patients were enrolled in the present study. Baseline characteristics of the study patients are summarized in Table 1. Of the 110 patients, 104 patients (95\%) met the criteria of IFN ineligibility. The reasons for IFN ineligibility are summarized in Table 2.

\section{Comparison of pretreatment factors between patients aged $\geq 75$ and $<75$ years}

The comparison of pretreatment factors between patients aged $\geq 75$ and $<75$ years is shown in Table 3 . There was a significant
Table 1. Baseline Characteristics of the 110 Patients in the Study

\begin{tabular}{|c|c|}
\hline Variable & Value \\
\hline Age, yr & $73(44-86)$ \\
\hline$\geq 75$ & $47(43)$ \\
\hline Sex, male/female & $52 / 58(47 / 53)$ \\
\hline Height, cm & $156.6(134.5-182.0)$ \\
\hline Weight, kg & $55.0(31.8-101.2)$ \\
\hline BMI, $\mathrm{kg} / \mathrm{m}^{2}$ & $22.3(12.5-36.8)$ \\
\hline IFN ineligible & $104(95)$ \\
\hline Cirrhosis & $76(69)$ \\
\hline Serious chronic kidney disease (eGFR<30) & $11(10)$ \\
\hline Hemodialysis & $8(7)$ \\
\hline History of HCC treatment & $41(37)$ \\
\hline History of IFN based therapy & $53(48)$ \\
\hline IFN intolerant & $11(10)$ \\
\hline NS3 RAVs positive & $18(16)$ \\
\hline D168E/D156S/Q80L/T54S & $6 / 1 / 8 / 3$ \\
\hline NS5A RAVs weakly positive & $7(6)$ \\
\hline Y93H/L31M+L31V & $5 / 2(5 / 2)$ \\
\hline Baseline HCV-RNA $\left(\operatorname{TaqMan}^{\circledR}\right), \log \mathrm{IU} / \mathrm{mL}$ & $6.1(2.6-7.2)$ \\
\hline $\mathrm{WBC}, / \mathrm{mm}^{3}$ & $4,220(1,000-10,470)$ \\
\hline Hemoglobin, g/dL & $12.9(8.6-16.7)$ \\
\hline Platelets, $\times 10^{4} / \mathrm{mm}^{3}$ & $12.4(5.0-30.2)$ \\
\hline AST, IU/L & $55(20-209)$ \\
\hline ALT, IU/L & $40(6-244)$ \\
\hline$\gamma-\mathrm{GT}, \mathrm{IU} / \mathrm{L}$ & $32(9-287)$ \\
\hline Type VI collagen 7S, ng/mL & $6.0(2.7-19.6)$ \\
\hline Hyaluronic acid, ng/mL & $275.5(25-4,547)$ \\
\hline AFP, ng/mL & $5.0(1.3-126.2)$ \\
\hline Creatinine & $0.37(0.39-10.33)$ \\
\hline
\end{tabular}

Data are presented as median (range) or number (\%).

BMI, body mass index; IFN, interferon; eGFR, estimated glomerular filtration rate; HCC, hepatocellular carcinoma; NS, nonstructural; RAVs, resistance-associated variants; HCV, hepatitis C virus; WBC, white blood cells; AST, aspartate aminotransferase; ALT, alanine aminotransferase; $\gamma$-GT, $\gamma$-glutamyl transferase; AFP, $\alpha$-fetoprotein.

Table 2. Reasons for Interferon Ineligibility

\begin{tabular}{lc}
\hline & No. (\%) \\
\hline Advanced age ( $\geq 65$ yr) & $82(75)$ \\
Other complications requiring medications* & $80(73)$ \\
Thrombocytopenia & $48(44)$ \\
Anemia & $39(35)$ \\
Neutropenia & $16(15)$ \\
Depression & $8(7)$ \\
\hline
\end{tabular}

*Hypertension, diabetes mellitus, autoimmune disease, thyroid disease, heart disease, renal disease, respiratory disease, and psychological disorder were included. 
Table 3. Comparison of the Pretreatment Factors between Patients Aged $\geq 75$ and $<75$ Years

\begin{tabular}{|c|c|c|c|}
\hline Factor & $\begin{array}{l}\text { Patients aged } \geq 75 \mathrm{yr} \\
\quad(\mathrm{n}=47)\end{array}$ & $\begin{array}{c}\text { Patients aged }<75 \mathrm{yr} \\
\qquad(\mathrm{n}=63)\end{array}$ & p-value \\
\hline Age, yr & 79 (75-86) & $65(44-74)$ & $<0.001$ \\
\hline Sex (male/female) & $19 / 28(40 / 60)$ & $33 / 30(52 / 48)$ & 0.250 \\
\hline Height, cm & $152.5(134.5-176.0)$ & $158.0(143.8-182.0)$ & 0.026 \\
\hline Weight, kg & $49.9 .9(37.3-79.2)$ & $58.2(31.8-101.2)$ & 0.002 \\
\hline BMI, $\mathrm{kg} / \mathrm{m}^{2}$ & $21.3(16.4-31.3)$ & $22.9(12.5-36.8)$ & 0.015 \\
\hline IFN ineligible & $47(100)$ & $57(90)$ & 0.032 \\
\hline Cirrhosis & $37(79)$ & $39(62)$ & 0.045 \\
\hline Serious chronic kidney disease $(\mathrm{eGFR}<30$ ) & $3(6)$ & $8(13)$ & 0.347 \\
\hline History of HCC treatment & $24(51)$ & $17(27)$ & 0.009 \\
\hline History of IFN based therapy & $20(43)$ & $33(52)$ & 0.339 \\
\hline IFN intolerant & $10(21)$ & $1(2)$ & 0.028 \\
\hline NS3 RAVs positive & $6(13)$ & $12(19)$ & 0.440 \\
\hline NS5A RAVs weakly positive & $3(6)$ & $4(6)$ & 1.000 \\
\hline Baseline HCV-RNA (TaqMan $\left.{ }^{\circledR}\right), \log$ IU/mL & $6.1(2.6-6.7)$ & $5.9(3.0-7.2)$ & 0.622 \\
\hline $\mathrm{WBC}, / \mathrm{mm}^{3}$ & $4,160(1,400-7,520)$ & $4,740(1,000-10,470)$ & 0.643 \\
\hline Hemoglobin, g/dL & $12.5(8.6-16.5)$ & $13.2(9.4-16.7)$ & 0.192 \\
\hline Platelets, $/ \mathrm{mm}^{3}$ & $12.3(4.6-22.3)$ & $13.3(2.5-29.5)$ & 0.228 \\
\hline AST, IU/L & $51(12-107)$ & $49(8-176)$ & 0.786 \\
\hline ALT, IU/L & $32(6-109)$ & $42(9-244)$ & 0.186 \\
\hline$\gamma$-GT, IU/L & $27(9-123)$ & $42(12-287)$ & 0.007 \\
\hline Type VI collagen 7S, ng/mL & $6.0(2.7-19.6)$ & $5.9(2.7-14.6))$ & 0.434 \\
\hline Hyaluronic acid, ng/mL & $282(26-4547)$ & $269(25-1,510)$ & 0.173 \\
\hline $\mathrm{AFP}, \mathrm{ng} / \mathrm{mL}$ & $4.9(1.3-126.2)$ & $5.0(1.5-118.3)$ & 0.749 \\
\hline Creatinine & $0.74(0.46-9.64)$ & $0.71(0.39-10.33)$ & 0.763 \\
\hline
\end{tabular}

Data are presented as median (range) or number (\%).

BMI, body mass index; IFN, interferon; eGFR, estimate glomerular filtration rate; HCC, hepatocellular carcinoma; NS, nonstructural; RAVs, resistance-associated variants; HCV, hepatitis C virus; WBC, white blood cells; AST, aspartate aminotransferase; ALT, alanine aminotransferase; $\gamma$-GT, $\gamma$-glutamyl transferase; AFP, $\alpha$-fetoprotein.

difference in height, weight, body mass index, IFN ineligibility, cirrhosis, history of HCC treatment, IFN intolerance and $\gamma$-GT levels.

\section{Safety and tolerability}

Adverse events profiles according to age group are summarized in Table 4. A similar safety profile was observed between patients aged $\geq 75$ and $<75$ years. The therapy of two patients (2\%) was discontinued due to adverse events. Reasons for discontinuation were hepatic failure with transaminase elevation in one of the patients aged $\geq 75$ years and severe dermatitis in one of the patients $<75$ years. Both discontinued patients achieved SVR. There was no significant difference in the frequency and severity of elevation of transaminase level between patients aged $\geq 75$ and $<75$ years.

\section{Treatment response}

Comparison of the viral negativity rate between patients aged $\geq 75$ and $<75$ years during treatment is shown in Fig. 2 . The viral negativity rate at week 4 of the patients aged $\geq 75$ years tended to be higher than that of patients $<75$ years $(p=0.054)$. SVR rates according to age groups are shown in Fig. 3. No significant difference was observed among the age groups. The SVR rates according to baseline factors are summarized in Table 5. The SVR rates in patients who had weakly positive $(<20 \%)$ NS5A RASs or chronic kidney disease (CKD) was 100\% (7/7) and 100\% (11/11), respectively.

\section{Treatment failure}

Viral relapse was shown in three patients (3\%) after treatment. All of them completed the treatment. Although one of them had pre-exiting NS3/4A RAS (A156S), they did not have pre-exiting NS5A RASs. Two patients achieved viral negativity at week 4 and one patient achieved it at week 8 after the start of therapy. Profiles of patients with treatment failure are summarized in Table 6. 
Table 4. Adverse Events during Treatment

\begin{tabular}{|c|c|c|c|}
\hline & $\begin{array}{l}\text { Patients aged } \geq 75 \mathrm{yr} \\
(\mathrm{n}=47)\end{array}$ & $\begin{array}{l}\text { Patients aged }<75 \mathrm{yr} \\
(\mathrm{n}=63)\end{array}$ & $\begin{array}{c}\text { Total } \\
(\mathrm{n}=110)\end{array}$ \\
\hline Treatment discontinuation due to adverse events & $1(2)$ & $1(2)$ & $2(2)$ \\
\hline Hepatic failure with transaminase elevation (grade 3) & 0 & $1(2)$ & $1(1)$ \\
\hline Dermatitis & $1(2)$ & 0 & $1(1)$ \\
\hline The other adverse events & $20(43)$ & $25(40)$ & $45(41)$ \\
\hline Elevation of transaminase level & $9(19)$ & $15(24)$ & $24(22)$ \\
\hline Grade 3 & $1(2)$ & 0 & $1(1)$ \\
\hline Grade 2 & $2(4)$ & $3(5)$ & $5(5)$ \\
\hline Grade 1 & $6(13)$ & $12(19)$ & $18(16)$ \\
\hline Elevation of serum ammonia level & $2(4)$ & $2(3)$ & $4(4)$ \\
\hline Pruritus & $3(6)$ & $1(1)$ & $4(4)$ \\
\hline Elevation of blood pressure & 0 & $1(1)$ & $1(1)$ \\
\hline Upper respiratory infection & 0 & $2(3)$ & $2(2)$ \\
\hline Mucositis oral & $1(2)$ & 0 & $1(1)$ \\
\hline Headache & $1(2)$ & $2(3)$ & $3(3)$ \\
\hline Hypertriglycemia & $1(2)$ & 0 & $1(1)$ \\
\hline Palpitations & $1(2)$ & 0 & $1(1)$ \\
\hline Fatigue & $1(2)$ & 0 & $1(1)$ \\
\hline Vertigo & $1(2)$ & 0 & $1(1)$ \\
\hline Insomnia & 0 & $2(3)$ & $2(2)$ \\
\hline
\end{tabular}

Data are presented as number (\%).

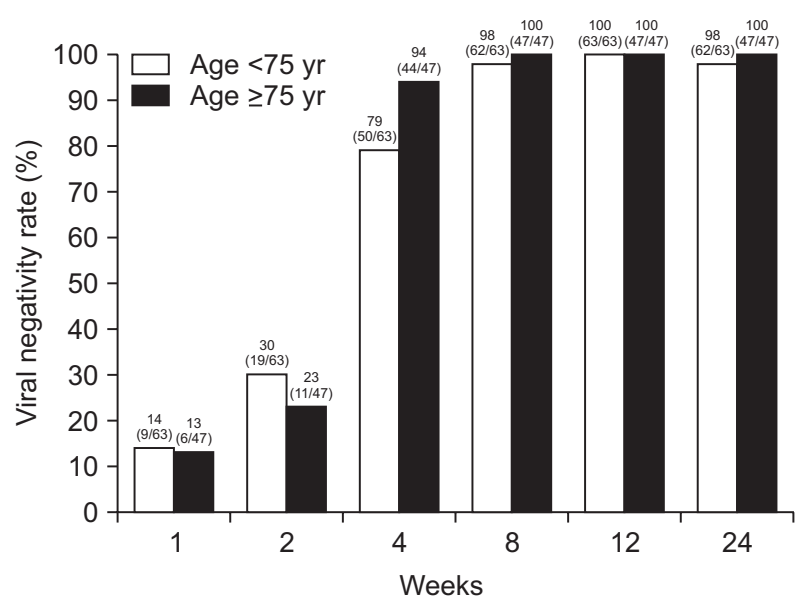

Fig. 2. A comparison of the viral negativity rate between patients aged $\geq 75$ and $<75$ years during treatment. The viral negativity rate at week 4 of patients aged $\geq 75$ years tended to be higher than that of patients aged $<75$ years $(\mathrm{p}=0.054)$.

\section{DISCUSSION}

We conducted a prospective cohort study of daclatasvir plus asunaprevir for mainly IFN-ineligible patients (95\%) in realworld settings. Our patients were older and had highly advanced fibrosis compared with those in previous clinical trials. ${ }^{8,10}$ In the present study, the median age was 73 years old; around 70\% of

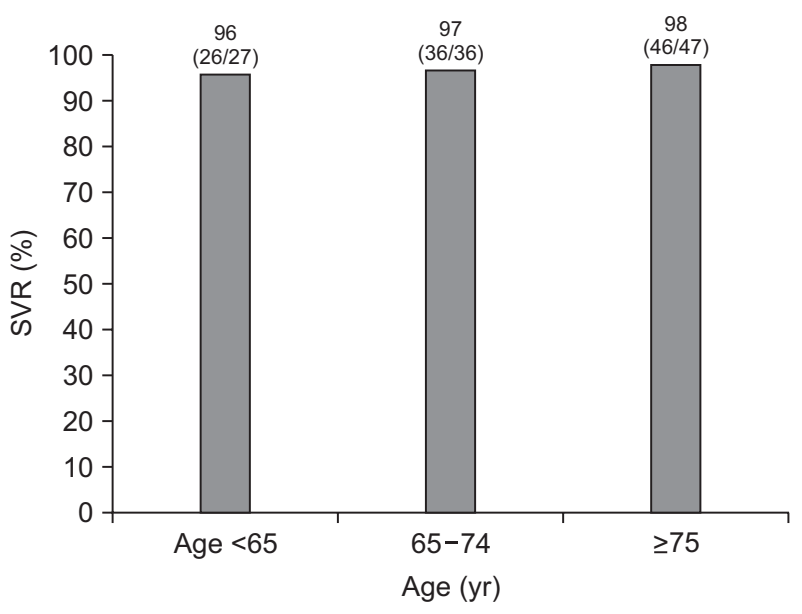

Fig. 3. The sustained virological response (SVR) rates according to age group. No significant differences were seen among the age groups.

the patients had cirrhosis and 73\% had comorbidities. Moreover, patients with serious renal dysfunction (10\%) or patients with a history of HCC treatment (37\%) were also included. Therefore, the present study could indicate real-world evidence for prevalent unmet medical needs in this area.

We showed that safety of the therapy in elderly patients over 75 years old was also very high and was comparable to that of 
patients under 75 years old. In previous Phase III trials, treatment discontinuation due to adverse events was low (1\% to $5 \%) .{ }^{8,10}$ The treatment discontinuation rate in the present study was also low (2\%). With regard to the safety of elderly patients over 75 years old, Ogawa et al. ${ }^{11}$ reported that there was no significant difference in the discontinuation rate due to adverse effects between the $<75$ and the $\geq 75$ years of age groups ( $4.6 \%$ vs $9.7 \%, p=0.09$ ). Morio et al. ${ }^{12}$ also reported that the frequency of adverse events was similar between the $<75$ and the $\geq 75$ years of age groups ( $2 \%$ vs $1 \%$ ). In other post-marketing studies in real-world clinical practice, the range of treatment discontinuation rate due to adverse events was at $2 \%$ to $15 \%$. $^{9,11,13,14}$ The reason for low discontinuation rate in our study may be attributed to scheduled prospective study with close monitoring. As treatment discontinuation has a high risk of treatment failure and strong RASs to multidrugs, close monitoring and adequate management of adverse effects are necessary.

Table 5. Sustained Virological Response Rates

\begin{tabular}{lc}
\hline & SVR rate \\
\hline Male & $100(52 / 52)$ \\
Female & $95(55 / 58)$ \\
Cirrhosis & $97(74 / 76)$ \\
Noncirrhosis & $97(33 / 34)$ \\
Age $\geq 65$ yr & $98(81 / 83)$ \\
Age $\geq 65$ yr and cirrhosis & $98(61 / 62)$ \\
IFN ineligible & $97(101 / 104)$ \\
IFN intolerant & $100(11 / 11)$ \\
Prior IFN treatment & $100(53 / 53)$ \\
No prior IFN treatment & $95(66 / 69)$ \\
Prior HCC treatment & $100(41 / 41)$ \\
No prior HCC treatment & $96(66 / 69)$ \\
Serious CKD (eGFR <30) & $100(11 / 11)$ \\
No serious CKD & $97(96 / 99)$ \\
NS3/4A RAVs positive & $94(17 / 18)$ \\
NS3/4A RAVs negative & $98(89 / 91)$ \\
NS5A RAVs weakly positive & $(7 / 7)$ \\
NS5A RAVs negative & $97 / 103)$ \\
\hline Nata & 97 \\
\hline
\end{tabular}

Data are presented as percent (number/total number). IFN, interferon; HCC, hepatocellular carcinoma; CKD, chronic kidney disease; eGFR, estimate glomerular filtration rate; NS, nonstructural; RAVs, resistance-associated variants.
The most frequent adverse event leading to discontinuation in previous trials was transaminase elevation. ${ }^{8,10}$ In the Japanese phase III trial, elevation of ALT level appeared in 16\% of the patients with 7\% of them having 3 to 4 grade abnormality. ${ }^{8} \mathrm{Al}-$ though the frequency of transaminase elevation in the present study was higher (22\%) when compared that in the Japanese phase III trial, the frequency of grade 3 to 4 abnormality was lower (2\%). In our study, as ursodeoxycholic acid administration was permitted as a treatment for drug-induced liver injury, the reason for low frequency of serious transaminase elevation may be attributed to the use of ursodeoxycholic acid. Nonetheless, further study is needed to verify the efficacy of ursodeoxycholic acid for asunaprevir-induced liver injury. Akuta et al. ${ }^{15}$ have reported that severe ALT elevations with asunaprevir concentrations tended to indicate higher rates and the discontinuation or reduction of asunaprevir improved ALT levels. To avoid the discontinuation of this treatment, the reduction of asunaprevir dose should be considered when ALT elevation appears during treatment.

In this study, despite the elderly patients of over 75 years old being smaller, having higher ineligibility and tolerance to IFN, being more cirrhotic, and having a higher rate of HCC treatment than those less than 75 years old, the efficacy was extremely high and the SVR rate approximated to 100\%. With regard to the efficacy of elderly patients over 75 years old, Ogawa et al. ${ }^{11}$ reported that the SVR rate of very old patients aged $(\geq 75$ years) was significantly lower than that of patients aged $>65$ years (90.8\% vs 98.5\%) despite patients with unfavorable factors affecting virological response, such as pre-existing NS5A RASs and prior simeprevir failure were excluded. The authors commented that the cause of the difference in the SVR rate was unknown. However, Morio et al. ${ }^{12}$ reported that the SVR rate for elderly patients over 75 years old was similar to that of younger patients (97\% and 92\%, respectively). Akuta et al. ${ }^{16}$ also reported that the SVR rates were $87 \%$ and $88 \%$ for all 844 patients and 411 elderly (>70 years of age), respectively. As mentioned, as the safety and efficacy of daclatasvir plus asunaprevir for elderly patients over 75 years old was similar to those in younger patients even in real-world settings, the use of this therapy should not be restricted by high age.

Background baseline factors such as age, sex, fibrosis status, IFN ineligibility and tolerance, prior IFN treatment, prior HCC treatment, serious CKD pre-existing NS3/4 RASs and weakly positive NS5A RASs had no effect on SVR. Morio et al..$^{13}$ indi-

Table 6. Profiles of Patients with Treatment Failure

\begin{tabular}{ccccccccc}
\hline Age, $y r$ & Sex & LC & History of IFN therapy & HCV-RNA, log IU/mL & Drug adherence & Pre-existing RAVs & RVR & RAVs at failure \\
\hline 52 & F & Yes & Naive & 6.4 & 100 & Negative & No & L31V, Y93H \\
74 & F & Yes & Naive & 6.0 & 100 & A156S & Yes & Not assessable \\
78 & F & No & Naive & 6.6 & 100 & Negative & Yes & D168E, Y93H \\
\hline
\end{tabular}

LC, liver cirrhosis; IFN, interferon; HCV, hepatitis C virus; RAVs, resistance-associated variants; RVR, rapid virological response; F, female. 
cated real-world evidence of efficacy and safety of daclatasvir plus asunaprevir in cirrhotic patients. The SVR rate of cirrhotic patients was similar to that of patients with chronic hepatitis (92.6\% vs 94.3\%). In the present study, the SVR rate of cirrhosis patients was 97\%. On the other hand, some reports have indicated the evidence of safety and efficacy for patients with CKD or hemodialysis patients. ${ }^{17-20}$ Our study also could show that the SVR rate of patients with serious CKD was 100\% (11/11). Hernandez et al. ${ }^{21}$ reported that SVR rates more than 95\% to daclatasvir plus asunaprevir were achieved to exclude patients infected with genotype $1 \mathrm{~b}$ with NS5A RASs at L31 or Y93H representing more than $20 \%$ of their virus population. Ikeda et al. ${ }^{22}$ also reported that the SVR rates with Y93H ratios of $<1 \%, 1 \%$ to $25 \%, 26 \%$ to $75 \%$ and $>75 \%$ were $99 \%, 100 \%, 71 \%$ and $23 \%$, respectively. In the present study, although patients with weakly positive NS5A RASs (<20\% of substitutions) were excluded as a general rule, their SVR rate was 100\% (7/7). Weakly positive NS5A RASs should have no effect on SVR. It can be considered that daclatasvir plus asunaprevir is suitable treatment for aged and/or cirrhotic patients even with serious CKD, a history of HCC treatment or weakly positive NS5A RASs.

Post-marketing studies have demonstrated that simeprevir failure and NS5A RASs (Y93 and L31 mutations) were significant unfavorable factors related to SVR. ${ }^{11,14,16}$ However, there were three patients with treatment failure in the present study in spite of the exclusion of NS5A RASs and simeprevir failure. Notably, all were relapse patients, had drug adherence of 100\% and had no history of IFN treatment and NS5A RASs. Rapid virological response (viral negativity at week 4) was achieved by two of the three patients. The reason for treatment failure was unknown from known pretreatment factors or treatment response. Uchida et al. ${ }^{23}$ reported that the SVR rate of daclatasvir plus asunaprevir for patients with NS5A-R30Q/H/L mutations at baseline was also lower than that of patients without NS5A RASs (77\% vs 95\%). In these patients, other minor NS5A RASs such as $\mathrm{R} 30 \mathrm{Q} / \mathrm{H} / \mathrm{L}$ might be associated with treatment failure.

There are some limitations in this study. First, as the patients without NS5A RASs and simeprevir failure were enrolled in this study, there were some selection biases. Second, the number of the patients is too small to conclude the safety and efficacy of elderly patients. Therefore, although early virological response rate of elderly patients tended to be higher, it may be just by chance. Third, the reason for the treatment failure of this dual therapy could not be clarified by our analysis. To validate our results and to clarify the reason for treatment failure, a largerscale cohort study and detailed analysis of RASs at baseline is needed. As far as we know, this dual therapy has been approved in only 17 countries of mainly Asian-Pacific region. This reason attributes to lower SVR rate and strong RASs to multidrugs after treatment failure. If this therapy can achieve the extremely high SVR rate (approximately 100\%) with high safety, it would be more cost-effective therapy than other expensive dual therapies such as sofosbuvir and ledipasvir in low-middle income countries.

In conclusion, the present study demonstrated that daclatasvir plus asunaprevir was a safe treatment even for patients over 75 years of age in real-world settings. When patients without preexisting NS5A RASs and prior simeprevir failure were selected, an extremely high SVR rate could be achieved irrespective of age. This treatment could be considered as one of cost effective options with obvious real-world evidence for patients ineligible for IFN, such as elderly patients with compensated cirrhosis and/or various comorbidities.

\section{CONFLICTS OF INTEREST}

No potential conflict of interest relevant to this article was reported.

\section{REFERENCES}

1. Asahina Y, Tsuchiya K, Tamaki N, et al. Effect of aging on risk for hepatocellular carcinoma in chronic hepatitis $\mathrm{C}$ virus infection. Hepatology 2010;52:518-527.

2. Editors of the Drafting Committee for Hepatitis Management Guidelines; The Japan Society of Hepatology. Guidelines for the management of hepatitis C virus infection: first edition, May 2012, The Japan Society of Hepatology. Hepatol Res 2013;43:1-34.

3. Tamai H, Shingaki N, Shiraki T, et al. Prediction of sustained response to low-dose pegylated interferon alpha-2b plus ribavirin in patients with genotype $1 \mathrm{~b}$ and high hepatitis $\mathrm{C}$ virus level using viral reduction within 2 weeks after therapy initiation. Hepatol Res 2011;41:1137-1144.

4. Tamai H, Mori Y, Shingaki N, et al. Low-dose pegylated interferon- $\alpha 2$ a plus ribavirin therapy for elderly and/or cirrhotic patients with HCV genotype-1b and high viral load. Antivir Ther 2014;19:107-115.

5. Kumada H, Toyota J, Okanoue T, Chayama K, Tsubouchi H, Hayashi N. Telaprevir with peginterferon and ribavirin for treatment-naive patients chronically infected with $\mathrm{HCV}$ of genotype 1 in Japan. J Hepatol 2012;56:78-84.

6. Hayashi N, Izumi N, Kumada H, et al. Simeprevir with peginterferon/ribavirin for treatment-naïve hepatitis $C$ genotype 1 patients in Japan: CONCERTO-1, a phase III trial. J Hepatol 2014;61:219227.

7. Tamai H, Shimizu R, Shingaki N, et al. Prediction of sustained virological response to telaprevir-based triple therapy using viral response within 2 weeks. Hepat Res Treat 2014;2014:748935.

8. Kumada H, Suzuki Y, Ikeda K, et al. Daclatasvir plus asunaprevir for chronic HCV genotype 1b infection. Hepatology 2014;59:20832091.

9. Kanda T, Yasui S, Nakamura M, et al. Daclatasvir plus asunaprevir treatment for real-world HCV genotype 1-infected patients in Japan. Int J Med Sci 2016;13:418-423. 
10. Manns M, Pol S, Jacobson IM, et al. All-oral daclatasvir plus asunaprevir for hepatitis $\mathrm{C}$ virus genotype $1 \mathrm{~b}$ : a multinational, phase 3, multicohort study. Lancet 2014;384:1597-1605.

11. Ogawa E, Furusyo N, Yamashita N, et al. Effectiveness and safety of daclatasvir plus asunaprevir for patients with hepatitis $\mathrm{C}$ virus genotype $1 \mathrm{~b}$ aged 75 years and over with or without cirrhosis. Hepatol Res 2017;47:E120-E131.

12. Morio R, Imamura M, Kawakami Y, et al. Safety and efficacy of dual therapy with daclatasvir and asunaprevir for older patients with chronic hepatitis C. J Gastroenterol 2017;52:504-511.

13. Morio K, Imamura M, Kawakami Y, et al. Real-world efficacy and safety of daclatasvir and asunaprevir therapy for hepatitis $\mathrm{C}$ virusinfected cirrhosis patients. J Gastroenterol Hepatol 2017;32:645650.

14. Iio E, Shimada N, Abe H, et al. Efficacy of daclatasvir/asunaprevir according to resistance-associated variants in chronic hepatitis $\mathrm{C}$ with genotype 1. J Gastroenterol 2017;52:94-103.

15. Akuta N, Sezaki H, Suzuki F, et al. Relationships between serum asunaprevir concentration and alanine aminotransferase elevation during daclatasvir plus asunaprevir for chronic HCV genotype $1 \mathrm{~b}$ infection. J Med Virol 2016;88:506-511.

16. Akuta N, Sezaki H, Suzuki F, et al. Favorable efficacy of daclatasvir plus asunaprevir in treatment of elderly Japanese patients infected with HCV genotype $1 \mathrm{~b}$ aged 70 and older. J Med Virol 2017;89:91-98.

17. Toyoda H, Kumada T, Tada T, et al. Safety and efficacy of dual direct-acting antiviral therapy (daclatasvir and asunaprevir) for chronic hepatitis $\mathrm{C}$ virus genotype 1 infection in patients on hemodialysis. J Gastroenterol 2016;51:741-747.

18. Suda G, Nagasaka A, Yamamoto Y, et al. Safety and efficacy of daclatasvir and asunaprevir in hepatitis $\mathrm{C}$ virus-infected patients with renal impairment. Hepatol Res 2017;47:1127-1136.

19. Suda G, Kudo M, Nagasaka A, et al. Efficacy and safety of daclatasvir and asunaprevir combination therapy in chronic hemodialysis patients with chronic hepatitis C. J Gastroenterol 2016;51:733740.

20. Nakamura Y, Imamura M, Kawakami Y, et al. Efficacy and safety of daclatasvir plus asunaprevir therapy for chronic hepatitis C patients with renal dysfunction. J Med Virol 2017;89:665-671.

21. Hernandez D, Yu F, Huang X, Kirov S, Pant S, McPhee F. Impact of pre-existing NS5A-L31 or -Y93H minor variants on response rates in patients infected with HCV genotype-1b treated with daclatasvir/asunaprevir. Adv Ther 2016;33:1169-1179.

22. Ikeda H, Watanabe $\mathrm{T}$, Okuse $\mathrm{C}$, et al. Impact of resistanceassociated variant dominancy on treatment in patients with HCV genotype 1b receiving daclatasvir/asunaprevir. J Med Virol 2017;89:99-105.

23. Uchida Y, Kouyama JI, Naiki K, et al. Development of rare resistance-associated variants that are extremely tolerant against NS5A inhibitors during daclatasvir/asunaprevir therapy by a twohit mechanism. Hepatol Res 2016;46:1234-1246. 